\title{
Structure of Cytochrome P450 2C9*2 in Complex with Losartan: Insights into the Effect of Genetic Polymorphism $₫$
}

\author{
Sonia J. Parikh, Chiara M. Evans, Juliet O. Obi, Qinghai Zhang, Keiko Maekawa, \\ Karen C. Glass, and Manish B. Shah \\ Department of Pharmaceutical Sciences, Albany College of Pharmacy and Health Sciences, Albany, New York (S.J.P., C.M.E., \\ J.O.O., K.C.G., M.B.S.); Department of Integrative Structural and Computational Biology, The Scripps Research Institute, \\ La Jolla, California (Q.Z.); and Department of Analytical Chemistry, Faculty of Pharmaceutical Sciences, Doshisha Women's \\ College of Liberal Arts, Kodo, Kyotanabe, Kyoto, Japan (K.M.)
}

Received April 13, 2020; accepted August 26, 2020

\section{ABSTRACT}

The human CYP2C9 plays a crucial role in the metabolic clearance of a wide range of clinical therapeutics. The * 2 allele is a prevalent genetic variation in CYP2C9 that is found in various populations. A marked reduction of catalytic activity toward many important drug substrates has been demonstrated by CYP2C9*2, which represents an amino acid variation at position 144 from arginine to cysteine. The crystal structure of CYP2C9*2 in complex with an antihypertensive drug losartan was solved using X-ray crystallography at $3.1-\AA$ resolution. The Arg144Cys variation in the *2 complex disrupts the hydrogen-bonding interactions that were observed between the side chain of arginine and neighboring residues in the losartan complex of CYP2C9 and the wild-type (WT) ligand-free structure. The conformation of several secondary structural elements is affected, thereby altering the binding and orientation of drug and important amino acid side chains in the distal active site cavity. The new structure revealed distinct interactions of losartan in the compact active site of CYP2C9*2 and differed in occupancy at the other binding sites previously identified in the WT-losartan complex.
Furthermore, the binding studies in solution using losartan illustrated lower activity of the CYP2C9*2 compared with the WT. Together, the findings yield valuable insights into the decreased hydroxylation activity of losartan in patients carrying CYP2C9*2 allele and provide a useful framework to investigate the effect of a single-nucleotide polymorphism that leads to altered metabolism of diverse drug substrates.

\section{SIGNIFICANCE STATEMENT}

The * 2 allele of the human drug-metabolizing enzyme CYP2C9 is found in different populations and results in significantly reduced activity toward various drug substrates. How the CYP2C9*2 variant induces altered drug metabolism is poorly understood given that the Arg144Cys variation is located far away from the active site. This work yield insight into the effect of distal variation using multitude of techniques that include X-ray crystallography, isothermal titration calorimetry, enzymatic characterization, and computational studies.
This work was supported by American Heart Association [Grant 19AIREA34450040] (to M.B.S.), 2019, and by the start-up funds from the Albany College of Pharmacy and Health Sciences (to M.B.S.). The work was supported by National Institutes of Health National Institute of General Medical Sciences [Grant R01-GM098538] (to Q.Z.) and from the Japan Society for the Promotion of Science [Grants-in-Aid for Scientific Research - KAKENHI Grant JP18K06613] (to K.M.). Use of Stanford Synchrotron Radiation Light (SSRL), Stanford Linear Accelerator Center - National Accelerator Laboratory, is supported by the US Department of Energy (DOE), Office of Science, Office of Basic Energy Sciences under Contract No. DE-AC02-76SF00515. The SSRL Structural Molecular Biology Program is supported by the DOE Office of Biological and Environmental Research and by the National Institutes of Health National Institute of General Medical Sciences [Grant P41-GM103393]. The contents of this publication are solely the responsibility of the authors and do not necessarily represent the official views of National Institute of General Medical Sciences or National Institutes of Health

https://doi.org/10.1124/molpharm.120.000042.

S This article has supplemental material available at molpharm. aspetjournals.org.

\section{Introduction}

Cytochrome P450 (P450)-dependent monooxygenases are a superfamily of heme-thiolate proteins involved in the metabolism of xenobiotics (Ortiz de Montellano, 2015). Of the 57 human P450 enzymes identified so far, about 17 enzymes are predominantly involved in the metabolism of clinical drugs (Guengerich et al., 2016). These include CYP3A4, CYP2D6, and CYP2C9, which are among the enzymes responsible for the clearance of majority of pharmaceuticals in phase I drug metabolism. Furthermore, the occurrence and frequency of genetic variations in P450 among different populations have a significant impact on enzyme function and variability in drug response (Sim and Ingelman-Sundberg, 2013). CYP2C9 metabolizes approximately $15 \%-20 \%$ of drugs, including the antihypertensive losartan, the anticoagulant warfarin, the analgesic ibuprofen, and the antidiabetic drugs tolbutamide and glimepiride (Rettie et al., 1992; Miners and Birkett,

ABBREVIATIONS: CHAPS, 3-[(3-cholamidopropyl)dimethylammonio]-1-propanesulfonic acid; CYMAL-5, 5- cyclohexyl-hexylpentyl- $\beta$-D-maltoside; ITC, isothermal titration calorimetry; PDB, Protein Data Bank; PMSF, phenylmethylsulfonyl fluoride; RMSD, root-mean-square deviation; TCEP, Tris (2-carboxyethyl) phosphine hydrochlorate; WT, wild type. 
1998; Yasar et al., 2001; Van Booven et al., 2010). The highly polymorphic nature of CYP2C9 has led to altered hydroxylation profiles of many substrates, resulting in a reduced ability to eliminate drugs in genetically affected individuals (Goldstein, 2001; Daly et al., 2017). CYP2C9*2 and CYP2C9*3 are two prevalent genetic variants that demonstrate significant reduction of catalytic activity compared with the wildtype (WT) enzyme, leading to therapeutic failure and adverse drug reactions (Rettie and Jones, 2005; Daly et al., 2017). These genetic variants have been studied functionally; however, the resulting amino acid change that occurs because of such variations and the distal location from the active site have made it difficult to infer their effect on enzyme activity. The *2 allele, which is found in approximately $15 \%$ of Caucasians and about 2\%-4\% of those of African or Asian descent, represents an amino acid change from arginine to cysteine at position 144 (Arg144Cys) (Crespi and Miller, 1997), whereas the CYP2C9*3 allele is found in up to $15 \%-20 \%$ of different populations and represents an amino acid change from isoleucine to leucine at position 359, or Ile359Leu (Sullivan-Klose et al., 1996).

The Protein Data Bank (PDB) lists more than 800 entries of the P450 structures from different species, with structures of over 100 human P450 isoforms in the presence or absence of ligands of various sizes and shapes (Berman et al., 2000). Despite significant progress in structural biology of P450 enzymes, details of the effect of genetic variations on the tertiary structure of the protein and the active site have remained limited. Our recent structural studies of CYP2C9 WT, CYP2C9*3, and CYP2C9*30 complexed with losartan illustrated the influence of single-nucleotide polymorphisms on the orientation of amino acid side chains in the active site (Maekawa et al., 2017). The effect of subtle differences in the amino acid substitution at a distal location can be transduced to the active site, thus directly affecting interactions with ligands in the binding pocket. Furthermore, considering the diverse substrate specificity of $\mathrm{P} 450$, such variations may lead to indirect effects on protein function in a substrate-dependent manner that are more difficult to predict. Overall, polymorphic variations can have local effects on interactions with neighboring residues and secondary structural elements as well as long-range conformational changes that alter the architecture of the active site and the access channel region important for ligand binding.

To further our understanding of how genetic variations in CYP2C9 affect drug binding and oxidation, we solved the $\mathrm{X}$-ray crystal structure of CYP2C9*2 in complex with the antihypertensive drug losartan and probed the binding of the drug using functional, biophysical, and computational approaches. The prodrug losartan is an angiotensin II receptor antagonist that is predominantly metabolized by CYP2C9 and CYP3A4 (Lo et al., 1995; Sica et al., 2005; Joy et al., 2009). The CYP2C9*2 structure in a conformationally distinct state was compared with the recently solved structures of CYP2C9 and provides insights into the implications of genetic polymorphisms on drug metabolism that would be useful in the development of drug candidates potentially effective to populations possessing such allele.

\section{Materials and Methods}

Materials. Tryptone enzymatic digest, yeast extract, glycerol, potassium phosphate dibasic, potassium phosphate monobasic, L-histidine monohydrochloride, ampicillin sodium salt, phenylmethylsulfonyl fluoride (PMSF), sodium chloride, EDTA, chloramphenicol, losartan potassium, Terrific broth, deoxyribonuclease I, and ribonuclease A were purchased from Sigma-Aldrich (St Louis, MO); Difco LuriaBertani Broth Lennox was purchased from Becton Dickinson (Franklin Lakes, NJ); isopropyl $\beta$-d-1-thiogalactopyranoside Dioxane-Free and 5-aminolevulinic acid hydrochloride were obtained from BOC Sciences (Upton, NY); 3-[(3-cholamidopropyl)dimethylammonio]-1propanesulfonic acid (CHAPS) and Tris(2-carboxyethyl)phosphine hydrochlorate (TCEP) were obtained from Soltec Venture (Beverly, MA); HisPur Ni-NTA (nitrilotriacetic acid) resin was obtained from ThermoFisher Scientific; and CM Macroprep was obtained from Bio-Rad (Hercules, CA). 5-Cyclohexyl-1-hexylpentyl- $\beta$-D-maltoside (CYMAL-5) was obtained from Anatrace (Maumee, OH). The $3 \alpha$-hydroxyl$7 \alpha, 12 \alpha$-di-(((2-(trimethylamino)ethyl)phosphoryl)ethyloxy)-cholane is a facial amphiphile used as described previously (Lee et al., 2013). PEGRx screens were obtained from Hampton Research. Human cytochrome $\mathrm{P} 450$ reductase was obtained from OriGene (Rockville, MD).

Protein Expression and Purification. The CYP2C9 WT cDNA was a gift from Dr. Keiko Maekawa. As described previously, the amino acid residues 1-23 (N-terminal transmembrane anchor domain) were replaced with amino acid residues MAKKT, and $\mathrm{C}$-terminal residue was substituted from $\mathrm{V}$ to I, which was followed by four histidine residues. The variant, CYP2C9*2 (Arg144Cys), was generated by site-directed mutagenesis using QuikChange II Site-Directed Mutagenesis Kit from Agilent and confirmed by sequencing. The plasmid was transformed into Escherichia coli Rosetta 2 cells. Protein expression and purification were carried out as described previously (Maekawa et al., 2017). A Luria-Bertani broth containing ampicillin $(100 \mu \mathrm{g} / \mathrm{ml})$ and chloramphenicol $(25$ $\mu \mathrm{g} / \mathrm{ml}$ ) was inoculated with the CYP2C9*2 plasmid glycerol stock and grown overnight at $37^{\circ} \mathrm{C}$ in an incubator shaker at $225 \mathrm{rpm}$. The Terrific broth was prepared, and $15 \mathrm{ml}$ of the overnight culture was inoculated into the medium supplemented with the Terrific broth salt, ampicillin, and chloramphenicol. The culture was further grown at $37^{\circ} \mathrm{C}$ with shaking at $230 \mathrm{rpm}$ until the optical density of a sample measured at a wavelength of $600 \mathrm{~nm}$ reached $0.7-0.8 \mathrm{~nm}$. The protein expression was induced by adding isopropyl $\beta$-d-1-thiogalactopyranoside $(1 \mathrm{mM})$ and 5 -aminolevulinic acid hydrochloride $(0.5 \mathrm{mM})$ and continued for 65 hours in the shaking incubator at $30^{\circ} \mathrm{C}$ and $190 \mathrm{rpm}$. Cells were harvested by centrifugation $(4000 \mathrm{~g}$ ) for 10 minutes.

The pellet was resuspended in $10 \%$ original culture volume in buffer (400 ml buffer for 4 liter culture) with $20 \mathrm{mM}$ potassium phosphate, $20 \%$ (v/v) glycerol, $1.25 \mathrm{mM}$ TCEP, and $1 \mathrm{mM}$ PMSF. The lysozyme $(0.3 \mathrm{mg} / \mathrm{ml})$ was added to the resuspended cells and stirred for 30 minutes prior to centrifugation at $5640 \mathrm{~g}$ for 30 minutes. The spheroplasts were resuspended in $5 \%$ original culture volume in $500 \mathrm{mM}$ potassium phosphate, $20 \%$ (v/v) glycerol, $1.25 \mathrm{mM}$ TCEP, $0.5 \mathrm{mM}$ PMSF, deoxyribonucelase $(10 \mu \mathrm{g} / \mathrm{ml})$, and ribonuclease A $(10 \mu \mathrm{g} / \mathrm{ml})$ and sonicated 40 pulses per minute for 1 minute for a total of 3 minutes. The detergent CHAPS was added to a final concentration of $0.8 \%$, and the lysate was stirred at $4^{\circ} \mathrm{C}$ for 90 minutes followed by centrifugation at $33,000 \mathrm{~g}$ for 1 hour.

The nickel resin was equilibrated using the buffer containing $50 \mathrm{mM}$ potassium phosphate and $20 \%(\mathrm{v} / \mathrm{v})$ glycerol $\left(\mathrm{pH} 7.4\right.$ at $\left.4^{\circ} \mathrm{C}\right)$. The protein supernatant was added to resin and was allowed to stir overnight at $4^{\circ} \mathrm{C}$ to facilitate protein binding. The mixture was centrifuged at $1840 \mathrm{~g}$ for 3 minutes to collect the resin with bound protein, which was then added to a Bio-Rad Econo-Column. Using the Bio-Rad NGC Quest chromatography system, the resin was washed with 10-column volume of the buffer containing $100 \mathrm{mM}$ potassium phosphate, $20 \%$ glycerol, $100 \mathrm{mM} \mathrm{NaCl}, 2 \mathrm{mM}$ TCEP, $0.5 \mathrm{mM}$ PMSF, $0.5 \%$ CHAPS, and $5 \mathrm{mM}$ histidine. The protein was eluted with $10 \mathrm{mM}$ potassium phosphate, $20 \%$ glycerol, $100 \mathrm{mM} \mathrm{NaCl}, 2 \mathrm{mM}$ TCEP, $0.5 \mathrm{mM}$ PMSF, $0.5 \%$ CHAPS, and $50 \mathrm{mM}$ histidine at a rate of $1 \mathrm{ml} /$ min. The absorbance at 415 and $280 \mathrm{~nm}$ was measured using UV spectrophotometer for each of the collected fraction, and the fractions 
with 415/280 $\mathrm{nm}$ greater than 1 were pooled before measuring the concentration. The pooled fractions were diluted five times by volume with the buffer containing $5 \mathrm{mM}$ potassium phosphate, $20 \%$ glycerol, $1 \mathrm{mM}$ EDTA, $2 \mathrm{mM}$ TCEP, $0.5 \mathrm{mM}$ PMSF, and 0.5\% CHAPS. The weak cation exchange resin, CM Macroprep (Bio-Rad), was equilibrated with $10 \mathrm{mM}$ potassium phosphate and $20 \%$ glycerol $(1 \mathrm{ml}$ resin/100 nmol protein), and the diluted protein was incubated overnight with the $\mathrm{CM}$ resin. The resin was collected by centrifugation at $2880 \mathrm{~g}$ and packed in a Bio-Rad Econo-Column before it was washed (10 times column volume) with the buffer containing $5 \mathrm{mM}$ potassium phosphate, $20 \%$ glycerol, $1 \mathrm{mM}$ EDTA, $2 \mathrm{mM}$ TCEP, and $50 \mathrm{mM} \mathrm{NaCl}$. The buffer containing $50 \mathrm{mM}$ potassium phosphate, $20 \%$ glycerol, $2 \mathrm{mM}$ TCEP, $1 \mathrm{mM}$ EDTA, and $500 \mathrm{mM} \mathrm{NaCl}$ was used to elute the protein at a rate of $1 \mathrm{ml} / \mathrm{min}$ in $1-\mathrm{ml}$ fractions. The absorbance at 415 and $280 \mathrm{~nm}$ was measured, and the fractions with $415 / 280 \mathrm{~nm}$ greater than 2 were pooled in a falcon tube on ice prior to measuring the concentration (Maekawa et al., 2017). The peak at $450 \mathrm{~nm}$ was visible in the presence of carbon monoxide and absent at $415-420 \mathrm{~nm}$. The CYP2C9 WT was expressed and purified in a similar manner.

Crystallization. The CM elution buffer was prepared by substituting $20 \%$ glycerol with $0.5 \mathrm{M}$ sucrose. The pooled protein sample was diluted to $18 \mu \mathrm{M}$ using the sucrose elution buffer $(50 \mathrm{mM}$ potassium phosphate, $500 \mathrm{mM} \mathrm{NaCl}, 0.5 \mathrm{M}$ sucrose, $1 \mathrm{mM}$ EDTA, $2 \mathrm{mM}$ TCEP). A stock solution of the drug losartan dissolved in water was prepared at 40-mM concentration, and losartan was added to a final concentration of $180 \mu \mathrm{M}$. The sample was incubated on ice overnight. Next day, the protein solution with the ligand was placed in the Amicon ultra concentrator (10 kDa mol. wt. cut-off) and concentrated via centrifugation to one-tenth volume followed by dilution to $18 \mu \mathrm{M}$ and addition of losartan to $180 \mu \mathrm{M}$. This process was repeated two times, and the protein-ligand complex was concentrated to a final concentration of $500 \mu \mathrm{M}$. The detergent CYMAL-5 was added to a final concentration of $4.8 \mathrm{mM}$ followed by the addition of $0.077 \%$ (w/v) 234-chol facial amphiphile. The sample was further supplemented by the addition of losartan ( $1 \mathrm{mM}$ final concentration) and filtered using the Amicon filtration tubes prior to crystallization. Crystal screening was carried out using sitting drop vapor diffusion by mixing screen solution (PEGRx 2 from Hampton Research) and CYP2C9*2-losartan complex in a $0.9: 1$ ratio. Plates were incubated at $18^{\circ} \mathrm{C}$ with a well reservoir volume of $50 \mu \mathrm{l}$. Crystals of CYP2C9*2 grew over the course of 1 to 2 weeks in drops containing $0.1 \mathrm{M}$ lithium sulfate monohydrate, $0.1 \mathrm{M}$ sodium citrate tribasic dehydrate ( $\mathrm{pH} 5.5$ ), and $20 \% \mathrm{w} / \mathrm{v}$ polyethylene glycol 1000. Crystals for the CYP2C9*2-losartan complex were collected in crystallization buffer with $20 \%$ sucrose as cryo-protectant and flashfrozen in liquid nitrogen. The crystals were shipped to Stanford Synchrotron Radiation Light source, and the crystallographic data were collected remotely using beam line 12-2 (Cohen et al., 2002; McPhillips et al., 2002; Soltis et al., 2008). Data were collected using $1^{\circ}$ oscillations ( 360 frames) and 5 -second exposure at $100 \mathrm{~K}$, and images were integrated using iMosflm and scaled using Scala (Leslie, 1999; Potterton et al., 2003; Evans, 2006; Winn et al., 2011).

Structure Determination and Refinement. The structure of CYP2C9*2 complex was solved at $3.1-\AA$ resolution. Briefly, the scaled data were used for molecular replacement using the automated program Balbes that used PDB 1OG2 as the template (Long et al., 2008). The solution was found in the space group $P 3_{1}$ with eight molecules (chains A to $\mathrm{H}$ ) in an asymmetric unit (Supplemental Fig. 1A). The Refmac5 was used for iterative refinement, and Coot was used for model building using the $2 F_{o}-F_{c}$ and $F_{o}-F_{c}$ electron density maps contoured to 1- $\sigma$ and $3-\sigma$, respectively (Emsley and Cowtan, 2004; Murshudov et al., 2011). The R-factor of 0.15 and R-free of 0.22 were achieved in the final refinement cycle. The losartan was modeled into the observed unbiased electron density near the active site heme prior to final steps of refinement (Supplemental Fig. 1B). Furthermore, an unidentified electron density was observed near the residues from 225 to 229 in each of the eight chains that was not modeled with any ligand in the final coordinates. The weak electron density near such ambiguous density was identified as the detergent CYMAL-5 as modeled. A total of six CYMAL-5 molecules were modeled in the structure individually located at the symmetry near the G-G' helix region and near residues 226-229 between the chains A-H as shown in Supplemental Fig. 1A. Furthermore, all the protein chains were identical [root-mean-square deviation (RMSD) $\sim 0.27 \AA$ ] to each other, and the chain A was used to generate the figures illustrated in this work. There were a total of 143 water molecules and three sulfate ions located in the structure. In addition, the following amino acid residue side chains, mostly lysine and glutamate, demonstrated disordered electron density in one or the other chains from $\mathrm{A}$ to $\mathrm{H}$ in the asymmetric unit: Lys28, Lys48, Lys52, Lys72, Glu81, Lys84, Glu92, Glu104, Lys118, Lys121, Arg132, Phe134, 135, Met136, 137, Lys138, Arg139, Asp143, Glu147, Arg150, Glu154, Lys160, Lys185, Lys190, Gln192, Glu199, Lys206, Lys235, Lys241, Glu246, Lys249, Glu253, Met257, Asn258, Gln261, Glu272, Lys273, Lys275, His276, Asn277, Gln278, Tyr283, Glu328, Arg333, Asn334, Gln340, Glu352, Lys375, Leu380, Lys383, Lys399, Met406, Asp414, Glu415, Lys421, Lys423, Lys459, Lys465, and Lys485. The final refinement statistics are presented in Supplemental Table 1. The coordinates and structure factors with a MolProbity score of $\sim 90$ th percentile (clash score for all atoms was 96th percentile) were submitted to the Protein Data Bank (PDB 6VLT) (Chen et al., 2010). All figures representing protein structures were created using PyMOL, The PyMOL Molecular Graphics System, Version 2.0, Schrodinger, LLC.

Active Site Volume Calculation and Ligand Docking. The volume of the active site of CYP2C $9 * 2$ was calculated using the computational software Voidoo using a probe occupied volume with a probe radius of $1.4 \AA$ (Kleywegt and Jones, 1994). All the solvent molecules, losartan, and CYMAL-5 were deleted from the coordinates of CYP2C9*2. The cavity search was performed using the coordinates in the region above the heme iron. AutoDock Vina was used to dock losartan into the current CYP2C9*2 complex (Trott and Olson, 2010). The losartan, CYMAL-5, waters and any other ligand or non-protein molecules except chain A were removed from the coordinate file, and docking was run using a rigid receptor molecule. The docking experiment included 20 events with a grid size of $50 \AA \times 50 \AA \times$ $50 \AA$ A centered on the heme iron.

Isothermal Titration Calorimetry. The ITC experiments were carried out by titrating CYP2C9 WT or CYP2C 922 with losartan at $25^{\circ} \mathrm{C}$ using a MicroCal ITC200 (GE Healthcare). The volume of the calorimetric cell was $202 \mu \mathrm{l}$, and the volume of the syringe was $40 \mu \mathrm{l}$. The purified CYP2C9 WT was dialyzed for 48 hours into buffer containing $50 \mathrm{mM}$ potassium phosphate ( $\mathrm{pH} 7.4), 500 \mathrm{mM} \mathrm{NaCl}, 1 \mathrm{mM}$ EDTA, and $1 \mathrm{mM}$ TCEP. Losartan (soluble in water) was diluted to its final concentration of $900 \mu \mathrm{M}$ using the dialysis buffer. Calorimetric titration experiments were performed by titrating losartan in the syringe at a concentration of $900 \mu \mathrm{M}$ into $30 \mu \mathrm{M}$ CYP2C9 or $100 \mu \mathrm{M}$ CYP2C9*2 protein in the ITC sample cell over 28 injections. The first injection of $0.5 \mu \mathrm{l}$ was followed by 27 injections at $1.4 \mu \mathrm{l}$ each, which were spaced apart by time intervals of 150 seconds after an initial delay of 180 seconds. The titration cell was continuously stirred at $750 \mathrm{rpm}$. The preliminary injection was excluded from integration and calculation of the $K_{D}$ values. The ITC binding isotherms were integrated using a Marquandt nonlinear least-squares analysis according to a one-set-of-sites binding model assuming a single set of identical binding sites with the Origin 7.0 program (OriginLab Corporation). First, the raw data were corrected for nonspecific heats of dilution determined by the magnitude of the peaks appearing after the system reaches complete saturation. The active fraction of the CYP2C9 enzyme in the calorimetric cell was calculated based on the $3: 1$ stoichiometric binding ratio of the WT and 1:1 ratio for the $* 2$ enzyme in complex with losartan observed in the crystal structure. The raw data were then integrated, normalized for concentration, and analyzed according to a one-set-of-sites binding model to calculate the binding affinity, the stoichiometry $(\mathrm{N})$, and the thermodynamic values. These experiments were repeated three times, and the overall method is consistent with the experiments performed previously with CYP2B6 (Wilderman et al., 2013). 
NADPH Consumption Assay. Reconstituted CYP2C9 assays were carried out in a final volume of $100 \mu$ l containing $50 \mathrm{mM}$ potassium phosphate buffer ( $\mathrm{pH} 7.4$ ) with 50 pmol CYP2C9 (WT or *2) incubated with 50 pmol human cytochrome $\mathrm{P} 450$ reductase on ice for 5 minutes (Bart and Scott, 2017). Twenty micromolars losartan was added, and the reaction was further incubated for 5 minutes at $37^{\circ} \mathrm{C}$. The reaction was initiated by the addition of $0.5 \mathrm{mM}$ NADPH. The reaction was immediately diluted to $1 \mathrm{ml}$ (in phosphate buffer), and absorbance was measured every 0.25 minutes for 10 minutes using ThermoScientific Biomate $160 \mathrm{UV}$-vis spectrophotometer. The rate of NADPH was calculated using an extinction coefficient of $6.22 \mathrm{mM}^{-1} \mathrm{~cm}^{-1}$ and expressed as nanomole NADPH consumed per minute per nanomole of CYP2C9.

Hydrogen-Peroxide Assay. The rate of hydrogen-peroxide formation was determined using the xylenol orange ion (III) colorimetric assay (Pierce, ThermoFisher Scientific) (Nourooz-Zadeh et al., 1994; Wei et al., 2007). Reactions were carried out in a final volume of $100 \mu \mathrm{l}$ with $50 \mathrm{mM}$ potassium phosphate buffer ( $\mathrm{pH} 7.4$ ), 50 pmol CYP2C9 (WT or *2), and $50 \mathrm{pmol}$ human cytochrome $\mathrm{P} 450$ reductase incubated on ice for 5 minutes. Losartan was added to a final concentration of $20 \mu \mathrm{M}$, and the mixture was incubated at $37^{\circ} \mathrm{C}$ for 5 minutes. The reaction was initiated by the addition of $0.5 \mathrm{mM} \mathrm{NADPH}$ and further incubated for $1,2,5,10$, and 30 minutes. Reactions were quenched by the addition of $30 \mu \mathrm{l}$ acetonitrile. Control samples were prepared without losartan, and blank was prepared without NADPH. Hydrogen-peroxide formation was detected by reading samples at $560 \mathrm{~nm}$, as outlined in the Pierce Quantitative Peroxide Assay Kit.

\section{Results}

Structure of CYP2C9*2 in Complex with Losartan (PDB 6VLT). The structure of CYP2C9*2 genetic variant complexed with losartan unraveled significant differences between the previously solved structure of the CYP2C9 WT in the presence of losartan (PDB 5XXI) and in the absence of any ligand (PDB 1OG2). In the crystal structure of the CYP2C9*2-losartan complex, the asymmetric unit is comprised of eight chains identical to each other, with an average RMSD of $\sim 0.27 \AA$. Electron density corresponding to losartan was consistent between all the chains, and one molecule of losartan was bound in the active site of each chain. Figure 1 shows location of the amino acid cysteine at position 144 on the D-helix, representing the genetic variation in CYP2C $9 * 2$ that is more than $15 \AA$ away from the active site where the losartan is bound. The losartan is oriented with a biphenyl ring closer to the heme iron, with the closest carbon atom of the ring at a distance of about $3.5 \AA$. The tetrazole ring is packed between the side chains of Glu300, Ile205, Phe476, and Thr301, as illustrated in Supplemental Fig. 2A. The imidazole ring of the losartan is facing the "roof" of the active site chamber, with the hydroxyl group making a polar contact with the nitrogen atom of asparagine side chain (Asn204). Furthermore, the guanidino group of arginine side chain at position 108 (Arg108), previously shown to be crucial for substrate coordination, interacts with the imidazole nitrogen of the drug molecule in the active site. The side chain of residue Phe 476 protrudes into the losartan access channel binding region found in the previously solved WT complex.

Importantly, a strong electron density consistent with binding of a ligand at the peripheral site was observed at the region near residues at positions 228 and 229 shown as $F_{o}-F_{c}$ map contoured at $3 \sigma$ in Supplemental Fig. 2B. This is similar to the site where the losartan molecule was bound on the surface at amino acid residues Phe226 to Thr229 in our

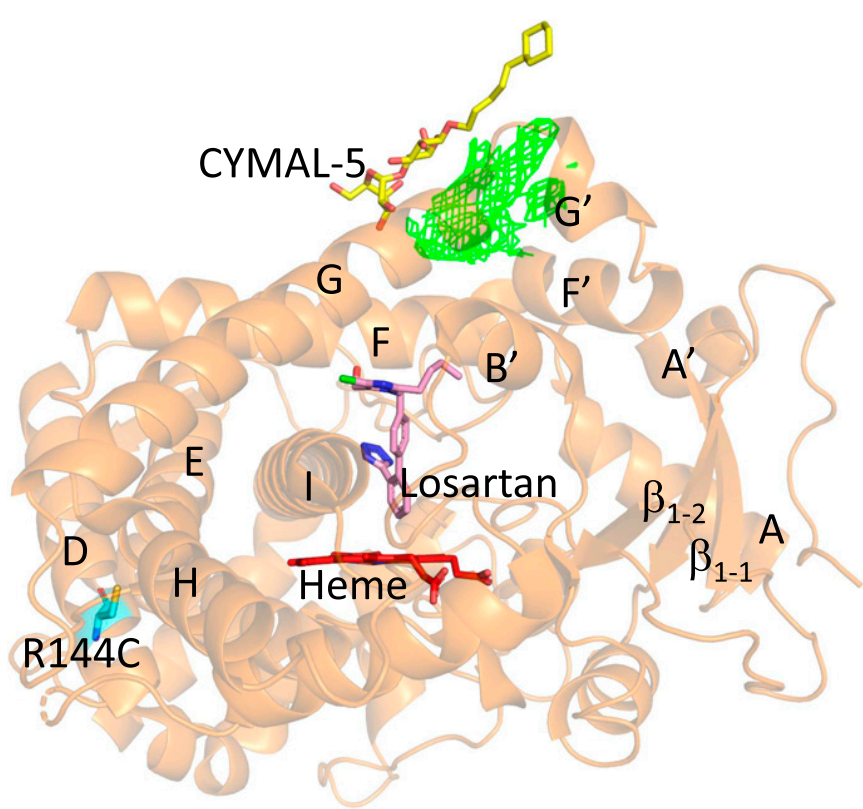

Fig. 1. Crystal structure of CYP2C $9 * 2$ in complex with the angiotensin II receptor antagonist drug losartan (shown in pink sticks) bound in the active site. The amino acid variation from arginine $(R)$ in the WT to cysteine (C) found in the current $* 2$ structure is illustrated as sticks in cyan color. The CYMAL-5 detergent molecule (yellow sticks) is shown bound near the electron density $\left(F_{o}-F_{c}\right.$ map in green mesh contoured to $3 \sigma$ ) of an unidentified ligand in the vicinity of the G-G' loop. Heme is shown as red sticks.

recently published structural complexes of CYP2C9 WT, CYP2C9*3, and CYP2C9*30 (Maekawa et al., 2017). However, the position and conformation of the density in the current structure, which is consistent among all the eight chains and also observed between symmetry-related molecules, is different from that in the previous complexes with losartan. Such differences in ligand binding could be attributed to the conformational changes transduced to this region from the distal genetic variation Arg144Cys in the *2 structure. In addition to the residues Gly228 and Thr229, this peripheral density also suggests continuous interaction with the side chain of Trp212. The weaker electron density in the new structure at relatively low resolution made it difficult to experimentally identify and model the losartan or ligand at this site consistent with the previous structures. Interestingly, adjacent to this less-clear electron density is the density that corresponds to CYMAL-5 as modeled in the structure, which indicates the role of detergent in crystal packing. Based on the $R$ and $R_{\text {free }}$ values for structure refinement and the improvement of electron density, losartan was a good possibility but not good enough to unambiguously model the drug. Notably, in the higher-resolution structural complexes with losartan that we published, the peripheral density was clear and unambiguous, thereby helping us experimentally model losartan. In addition to considering the components in the crystallization buffer, modeling facial amphiphile [3 $\alpha$ hydroxyl- $7 \alpha, 12 \alpha$-di-(((2-(trimethylamino)ethyl)phosphoryl) ethyloxy)-cholane] used during crystallization was also attempted without any success.

Comparison with CYP2C9 WT-Losartan (PDB 5XXI). The structural differences between the current CYP2C9*2losartan complex and the recently published structure of 


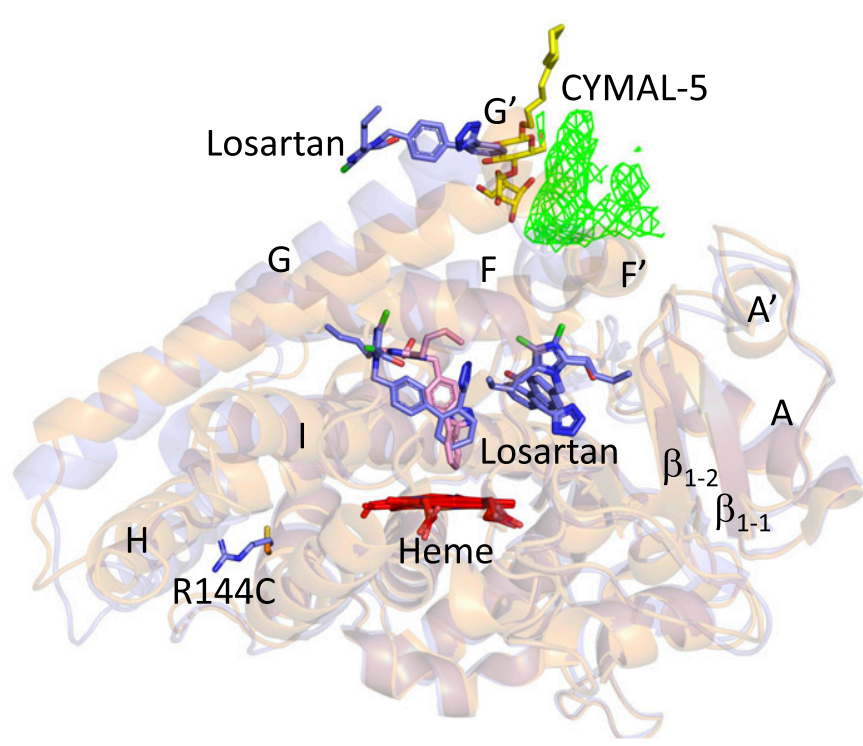

Fig. 2. CYP2C9*2 (brown) bound to losartan (pink sticks) is superimposed to previously solved CYP2C9 WT-losartan complex (violet). The structural overlay clearly illustrates the differences between the two structures bound to the same drug across several secondary structural elements. Additional important difference includes binding of three losartan molecules with the WT vs. one with the *2 complex and the unmodeled electron density (green mesh) of a ligand located near the peripheral losartan binding site. The CYMAL-5 in the *2 complex near this density is shown in yellow sticks. The heme is shown in red stick representation.

CYP2C9 WT in complex with losartan are noteworthy and pronounced. The structural overlay of the CYP2C9 WTlosartan complex and the new CYP2C9*2-losartan complex revealed the RMSD of $\sim 0.75 \AA$ with a large difference between the F-G cassette in the $* 2$ structure, suggesting a possible role for the distal amino acid (arginine to cysteine or Arg144Cys) as shown in Fig. 2. The marked shift across several residues along with the distinct orientations of side chains was observed in the F, F', G', and G helices, which also affects the active site. The residue side chains located within $5 \AA$ of losartan in both of the complexes are shown in Supplemental Fig. 3. Furthermore, the CYP2C9 WT in complex with losartan bound in the active site reveals that the access channel is remarkably more open than in the structure of *2 in complex with losartan. Importantly, the guanidino group of the side chain of Arg144 on the D-helix makes polar contact with the main chain oxygen atom of Gln261 on the G-H loop in the WT-losartan complex (Fig. 3). The variation Arg144Cys in *2 disrupts such hydrogen-bonding interactions between these secondary structural elements that were "bridged" together in the WT, and may lead to long-range rearrangements in the F-G cassette making the *2 structure more compact, with only one losartan bound compared with two losartans in the WT complex. This is further reflected in the differences in orientation of losartan in the active site between the two structures. As illustrated in Fig. 4A, the losartan reorients in the $* 2$ structure and is pushed further into the active site, with the closest atom of the biphenyl ring from the heme iron at $\sim 3.7 \AA$ compared with $\sim 4.7 \AA$ in the WT. The tetrazole of losartan is also rotated by almost $90^{\circ}$ in the compact active site, with the imidazole group reorienting to fit in a horizontal fashion compared with its vertical orientation in the WT complex. The alkyl chain that extends toward the F helix in the WT complex is now located in the alternate direction near the B' helix region. Similar structural perturbations were observed upon comparing the current CYP2C9*2 with the previously solved CYP2C9*3-losartan complex because the *3 and WT-losartan complexes were superimposable. However, the orientation of losartan in the active site and the total number of losartans bound differed in the *2 and *3 structures.

Compared with the WT-losartan complex, the residue side chains from Trp212 to Lys235 on the F-G cassette demonstrated altered conformation in the $* 2$ complex that could have a potential impact on ligand binding and interaction (Supplemental Fig. 4). This also includes the region of losartan binding at the surface or peripheral site from residues Phe226 to Thr229, as observed in the WT as well as *3- and *30-losartan complexes and the disordered or ambiguous electron density seen in *2 bound with losartan. Such changes in conformation of amino acid side chains could be influenced by structural rearrangements due to the longrange effect of the distal amino acid variation. The absence of an additional molecule of losartan located in the access channel of the WT complex with easy access to the solvent is likely due to the movement of the F-G cassette that shifts closer to the A' helix leading to the closure of the entrance near the surface in the $* 2$ complex. This also leads to the rotation of amino acid side chains, particularly Phe69, which reorients more than $90^{\circ}$ to avoid clashes with the F'-G' helices (Fig. 4B). The guanidino side chain of Arg108 that illustrated hydrogenbonding interactions with the tetrazole ring and the hydroxyl of the imidazole ring in the WT complex now reorients and interacts with the imidazole nitrogen of losartan in this conformation (Fig. 4B). The Phe476 side chain on the $\beta_{4}$ loop protrudes into the access channel in a similar fashion, as observed in the *3-losartan complex near the binding site of third losartan molecule that was only observed in the WT and *30 complexes (Maekawa et al., 2017). The difference in the presence or absence of such a channel near the binding site of third losartan molecule was confirmed by MoleOnline software (unpublished data), which clearly illustrated the access

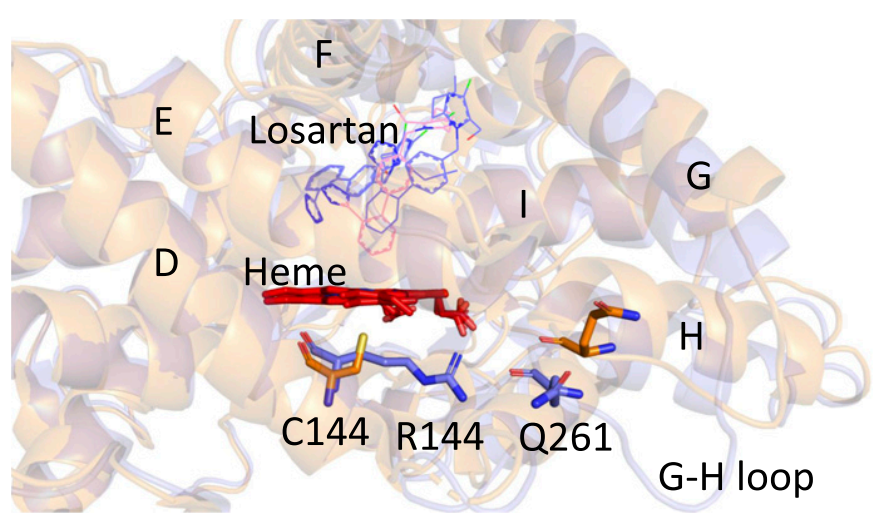

Fig. 3. The overlay of CYP2C9*2 (brown) in complex with losartan with the CYP2C9 WT-losartan complex (violet) in an orthogonal representation focusing on distal variation. The side chain of arginine (R144 or Arg144) in the WT complex, the side chain of cysteine (C144) in the *2 complex, and the side chain of glutamine at 261 (Q261) are shown in stick representation. The differences in the G-H loop region are observed that extends to the F-G cassette. Heme is shown in red sticks, and the active site losartan is shown in thin lines. 


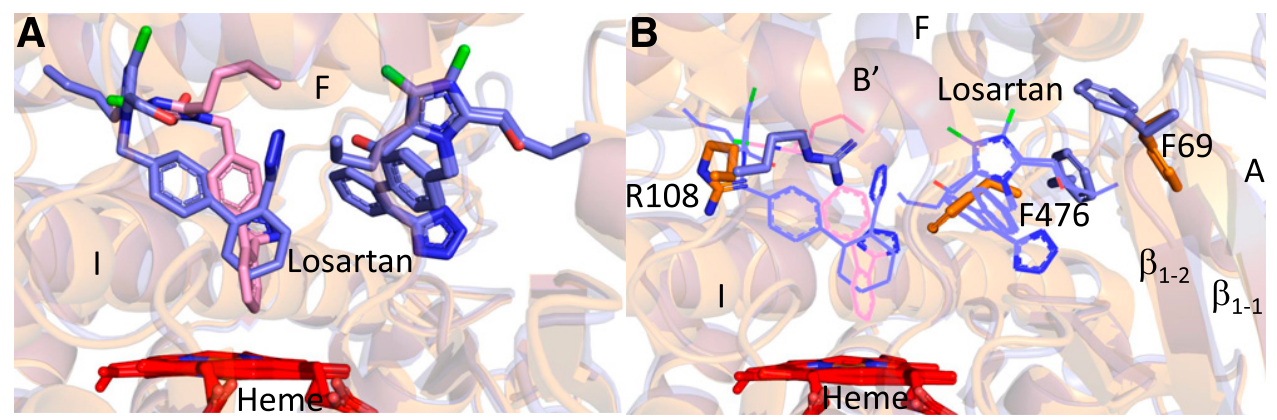

Fig. 4. Structural overlay of the CYP2C9*2-losartan complex (brown) and the CYP2C9 WT-losartan complex (violet). (A) The overlay demonstrates the altered orientation of losartan (pink sticks) in the *2 complex in the compact active site and the multiple molecules of losartan (violet sticks) bound to the WT. The panel (B) shows the changes in orientation as well as interaction of crucial Arg108 side chain, which interacts with the imidazole nitrogen in the *2 complex compared with the interaction with the tetrazole in the WT complex. Moreover, the Phe476 side chain in the *2 complex is shown protruding near the access channel binding site of losartan in the WT complex. Another crucial difference includes the rotation of Phe69 side chain on the A helix likely due to the movement of the F' and G' helices in the *2 complex.

from the surface in the WT but not the $* 2$ complex (Pravda et al., 2018).

Structural Comparison with CYP2C9 WT Ligand-Free (PDB 10G2). Comparing the CYP2C9*2 losartan bound structure with the previously solved WT ligand-free structure revealed notable difference in the F-G cassette consistent with that observed with the CYP2C9 WT-losartan complex (Fig. 5A) (Williams et al., 2003). The Arg144Cys variation on the D-helix illustrated local effects in amino acid interactions, suggesting that such changes propagate to the active site through the long-range conformational changes in the F-G region. As shown in Fig. 5B, the side chain of Arg144 makes a polar contact with the main chain oxygen atoms of His184 on the E helix and Gln261 on the G-H loop in the WT ligand-free structure. The cysteine variation at 144 likely affected such "conserved" interactions with the G-H loop, indicating the importance of such interactions in structure and function. The loss of polar contacts with Arg144 may have contributed to displacement of the amino acids His184 (E helix region) and Gln261 (G-H loop region), which are now located further away in the CYP2C $9 * 2$ complex compared with the ligand-free structure. Despite the modest effect on the overall conformation of $\mathrm{E}$ and $\mathrm{H}$ helices, the G-H loop and the adjoining F-G cassette showed much larger and significant differences in conformation of F, F', G', and G helices and the associated loop regions between the two structures. Such conformational perturbations may have consequently resulted in the more compact active site observed in the $* 2$ complex than in the WT ligand-free structure. Notably, several amino acids on the C-D loop ( 135-140) near the Arg144 region are not consistently observed in many P450 structures because of disorder in electron density. Thus, this loop region is often not modeled with amino acids in many P450 crystal structures because of disordered or missing electron density resulting from the crystallographic data (Shah et al., 2011, 2016). This was consistent in the current CYP2C9*2 complex in which the residues from 135 to 138 were not modeled because of disorder in several chains. Furthermore, this C-D loop region in the P450 enzymes has been previously shown to be the binding site with the NADPH-dependent cytochrome $\mathrm{P} 450$ reductase crucial for electron transfer to the heme iron for substrate hydroxylation (Bumpus and Hollenberg, 2010).

Additionally, the F' and G' helices located at the access channel region and $\mathrm{F}$ and $\mathrm{G}$ helices that border the active site include several amino acids known to interact with the substrates. The engineering of multiple amino acids in the F' and G' helices (seven residues between 206 and 224) near the tip of the access channel in the WT ligand-free structure made it difficult to clearly interpret the difference in the orientation of amino acid side chains in that region (Williams et al., 2003). The other secondary structural elements that exhibit considerable differences include the I-helix and the $\beta_{4}$ loop. The I-helix that forms the "wall" of the active site, with residues located in close proximity to losartan, illustrated movement likely due to the packing of the tetrazole moiety in the region. The $\beta_{4}$ loop that extends near the access channel in the $* 2$ complex displayed considerable differences in the structural overlay, with the side chain of Phe 476 rotating by $90^{\circ}$ and protruding into the active site compared with the WT (Fig. 5C). Moreover, in the WT ligand-free structure, the side chain of Arg108 is rotated out of the active site and toward the surface rather than interacting with losartan in the current structure. Supplemental Figure 5 shows an overlay comparing the position of active site residues located within $5 \AA$ of the losartan in the two structures.

Active Site Cavity Volume and Computational Docking of Losartan. The active site cavity volume $\left(\sim 1000 \mathrm{~A}^{3}\right)$ of the CYP2C9*2-losartan complex (PDB 6VLT) was elucidated using Voidoo and compared with the previously solved structure of the CYP2C9 WT-losartan complex (PDB 5XXI) (Kleywegt and Jones, 1994). The structural overlay clearly illustrates the reduced active site volume leading to the compact binding pocket of the $* 2$ complex (Fig. 6A). The movement of the F-G cassette and the binding and orientation of losartan in the binding pocket contributed to the differences observed in the architecture and volume of the active site compared with the WT complex. In addition to the movement of several secondary structural elements, including a major change in the rotation of the Phe476 side chain, which protrudes in the access channel region at the periphery of the active site significantly limiting the volume of the cavity. The overall structural changes observed in the $* 2$ complex along with such reorientation of amino acid side chains contributed to a closed binding pocket with just one molecule of losartan bound compared with the much more open and accessible pocket found in the WT complex. The open conformation of the CYP2C9 WT-losartan complex with an additional ligand-binding site in the access channel demonstrated 
A

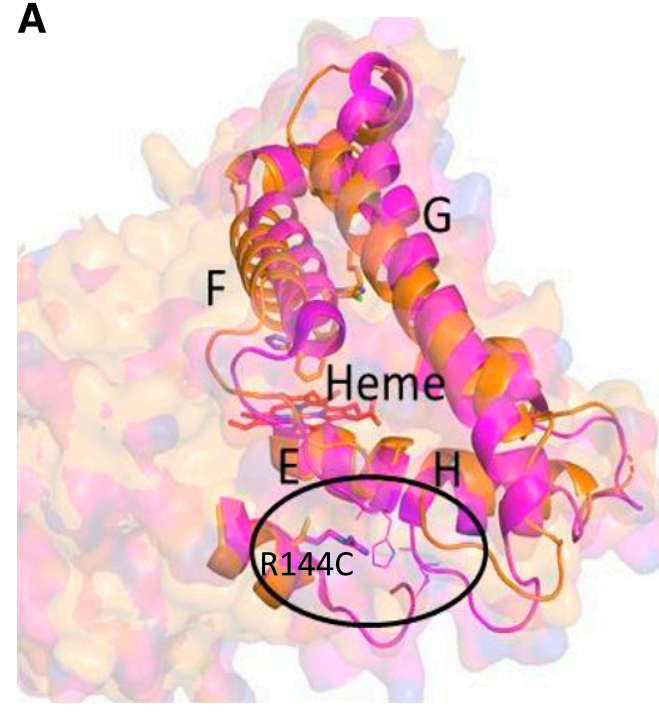

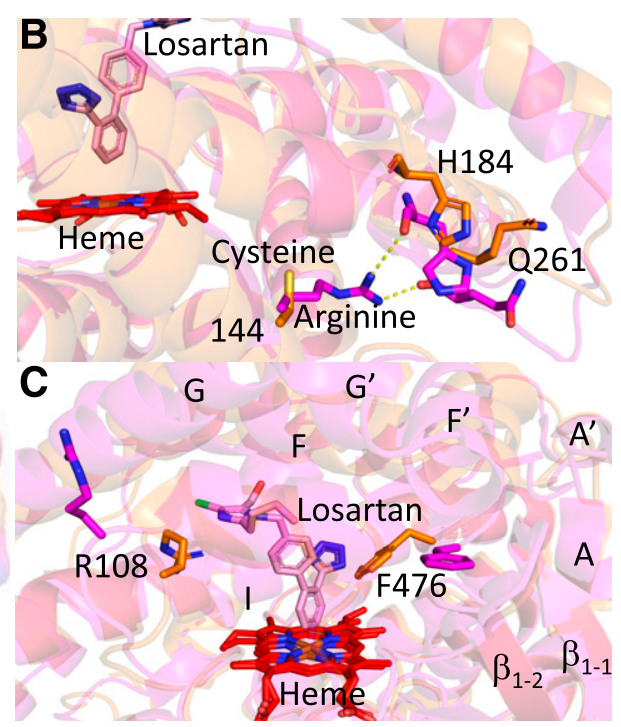

Fig. 5. Structural overlay of CYP2C $9 * 2$ losartan (brown) and CYP2C9 WT (magenta) ligand-free. Heme (red), losartan (pink), and amino acid side chains are shown in stick representation. (A) The location of Arg144Cys change is represented with amino acid side chains as marked near the $\mathrm{E}$ and the G-H loop in the overlay. (B) The hydrogen-bonding interaction between the side chain of Arg144 and the main chain atoms of His184 and Gln261 on the neighboring $\mathrm{E}$ and G-H loop, respectively, observed in the ligand-free structure of CYP2C9 WT is absent in the $* 2$ or 144 Cys variant complex. (C) The differences of the amino acids Arg108 in the active site and Phe476 near the access channel are shown, with the side chains reorienting or protruding the active site in the $* 2$ complex. a significant "leaking effect," in which the active site cavity extends to the solvent region or surface of the protein (Cuff and Martin, 2004). Thus, despite several attempts it was not possible to accurately calculate the active site cavity volume of the WT-losartan complex in PDB 5XXI.

Computational ligand docking was performed using AutoDock Vina to explore the utility of the newly available CYP2C9*2 structure in predicting substrate interactions and investigate the binding orientation of losartan in the active site (Trott and Olson, 2010). Since the *2 crystal structure demonstrated an unproductive orientation of losartan in the active site, computational docking helped to illustrate that losartan could adopt the preferred or productive mode that is consistent with the previously illustrated metabolism in the active site. The docked losartan was able to adopt multiple poses in the active site of CYP2C9*2, and the highest affinity pose placed losartan with the imidazole ring with hydroxyl oriented toward the heme iron in the active site (Fig. 6B). Such an orientation would be consistent with the demonstrated site of metabolism in losartan by CYP2C9. The following two highest affinity poses in the active site included either the alkyl chain closest to the heme iron or the phenyl ring near the heme iron similar to that observed in the crystal structure. Docking utilizing the previously solved CYP2C9 WT (PDB 5XXI or losartan after removing ligands from the coordinates) as a receptor produced a variety of poses of losartan. The one with highest affinity had the phenyl ring oriented toward the heme iron in the active site, which is consistent with that observed in the actual CYP2C9 WT-losartan structure. Those with the imidazole ring or the alkyl chain near the heme iron were also among the high affinity poses observed with the WTlosartan structure, further suggesting that losartan could adopt multiple conformations or productive mode in the active site.

Functional Characterization of Losartan Binding to CYP2C9*2. Isothermal titration calorimetry was employed to probe the binding affinity of losartan in solution by titrating with the CYP2C9 WT and CYP2C9*2 variant. The CYP2C9*2 and losartan interaction illustrated marked reduction in binding compared with the WT. The average $K_{D}$ value for losartan binding to CYP2C9*2 was $9 \pm 0.5 \mu \mathrm{M}$, which is weaker than the $K_{D}$ value of $6.8 \pm 0.7 \mu \mathrm{M}$ yielded by the WT enzyme (Fig. 7). The activity of the enzyme was measured in the reconstituted system containing the redox partner

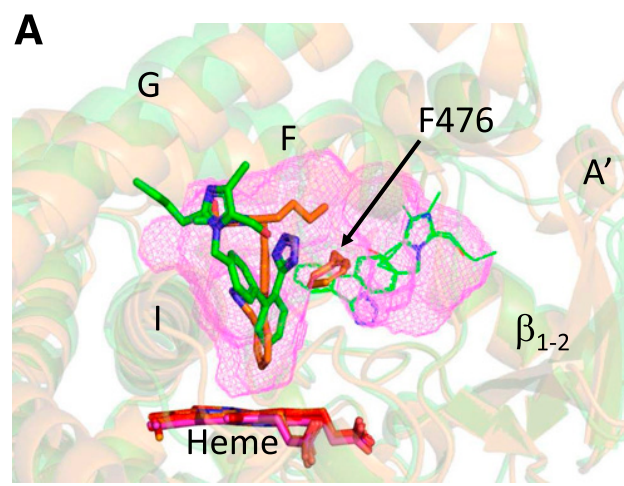

B

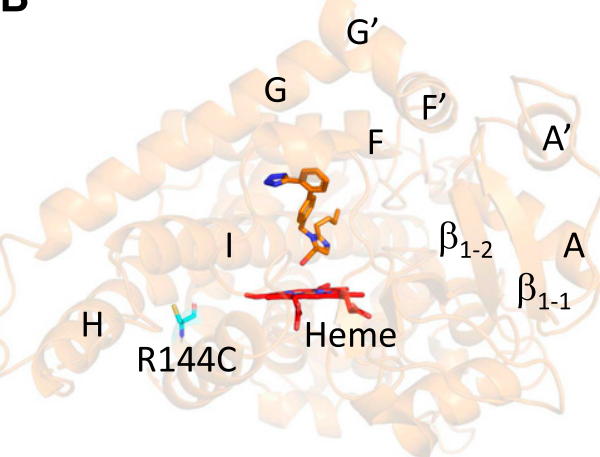

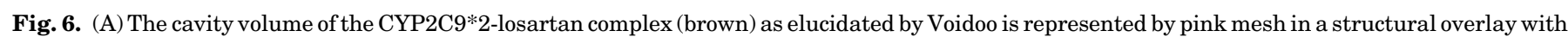

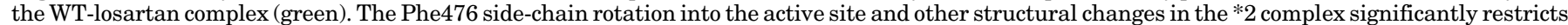

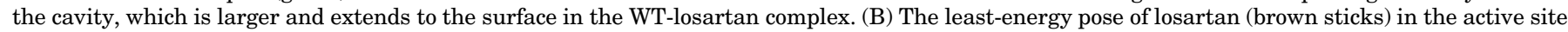

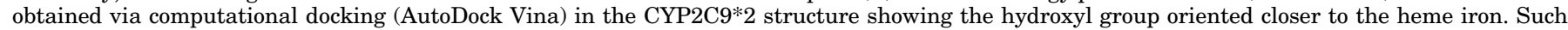
orientation would be favored or consistent to the demonstrated metabolism of losartan. 
cytochrome $\mathrm{P} 450$ reductase and substrate losartan by the consumption of NADPH. The rate of $\mathrm{NADPH}$ used by cytochrome $\mathrm{P} 450$ reductase was determined by the change in absorbance at $340 \mathrm{~nm}$ as a function of time. The recombinant CYP2C9 WT incubations resulted in an NADPH depletion rate that was markedly higher than the same noted for the CYP2C9*2, indicating the reduced ability of the variant enzyme to turn over substrate losartan (Fig. 8A). Furthermore, the hydrogen-peroxide formation was monitored in the presence of substrate losartan in an assay system using xylenol orange reagent. The hydrogen-peroxide formation dropped with CYP2C9*2 compared with the WT enzyme as shown in Fig. 8B. The reduced binding affinity and activity of the CYP2C9*2 enzyme compared with those of the WT toward losartan are consistent with the structural studies that illustrate the long-range effects of the genetic variation occurring far away from the active site. Both functional assays were repeated in triplicate, and S.D. was expressed as error bars.

\section{Discussion}

The Arg144 residue is located in close proximity to the region demonstrated to be involved in the interaction between P450 and its electron donor NADPH-dependent cytochrome $\mathrm{P} 450$ reductase, suggesting that the Arg144Cys variation may primarily affect the interaction with its redox partner (Crespi and Miller, 1997; Bumpus and Hollenberg, 2010). This is consistent with the significantly decreased $V_{\max }$ for CYP2C9*2 variant, indicating reduced efficiency for substrate turnover, which may be attributed to the altered interaction with the D-helix where residue 144 resides (Ho et al., 2003; Niinuma et al., 2014). However, this does not discount the remarkable changes seen in the active site configuration of CYP2C9*2 compared with the WT structure since another study noted a connection between coupling and uncoupling of products in reduced catalytic activity (Wei et al., 2007). The CYP2C9 WT complex illustrated three molecules of losartan bound: one in the active site, another in the access channel, and third on the surface near F'-G' helices. However, the *2 complex was clearly devoid of the second molecule in the access channel and demonstrated disordered electron density for a ligand not modeled near the F'-G' helices on the surface. The genetic variation Arg144Cys at the C-D loop region appears to alter the F-G cassette at several important amino acids that may have contributed to the difference in binding and orientation of losartan in the active site as well as the absence of losartan in the access channel. The absence of losartan binding in the access channel is similar to that observed with the previously solved CYP2C9*3-losartan structure. Additionally, the movement of the $\beta_{4}$ loop and the reorientation of the crucial Phe 476 side chain protruding in the access channel in the *2 and *3 complex clearly affect the losartan binding in the access channel of these structures compared with the WT. On the contrary, the losartan was bound in both the access channel and the active site, the CYP2C9*30 complex (PDB 5X23), which showed significantly reduced activity toward losartan and other substrates in multiple studies (Maekawa et al., 2009; Niinuma et al., 2014). The Phe476 side-chain orientation in this structure is consistent with what is observed in the WT complex. Supplemental Figure 6 clearly shows the differences in the positioning of the Phe476 side chain observed in these three important genetic variants compared with the WT structure in complex with the clinical drug substrate losartan. As shown previously, the Gln214 side chain in the WT complex interacts with the imidazole ring of the access channel losartan near the hydroxyl moiety, which is the potential activation site of losartan. The rotation of Gln214 side chain in *30, and its hydrogen bonding with the hydrophilic threonine substitution in the Ala477Thr amino acid variation may have played a role
A

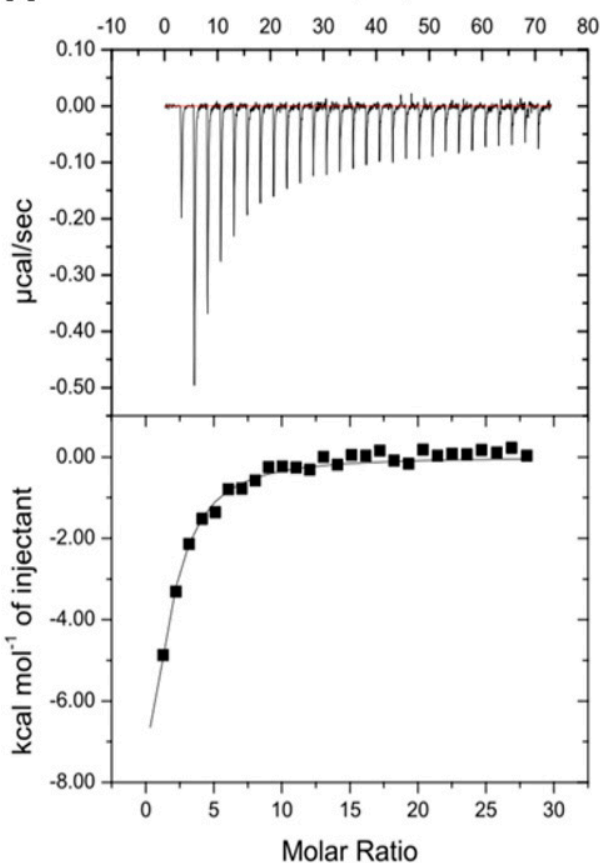

B

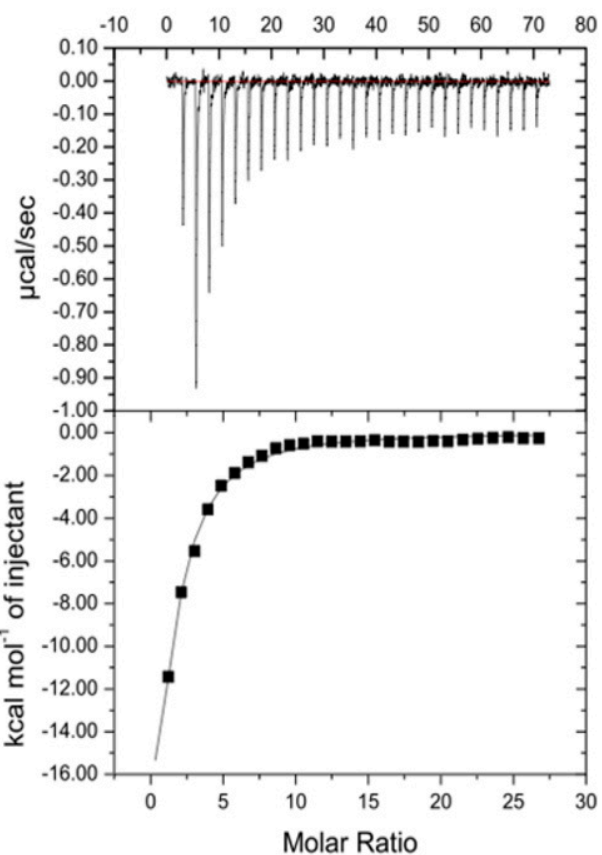

Fig. 7. The ITC binding isotherms for CYP2C9 WT (A) and CYP2C9*2 (B) with losartan. The ligand-binding titration assays were performed in triplicate, and the binding affinities with S.D. are reported. 


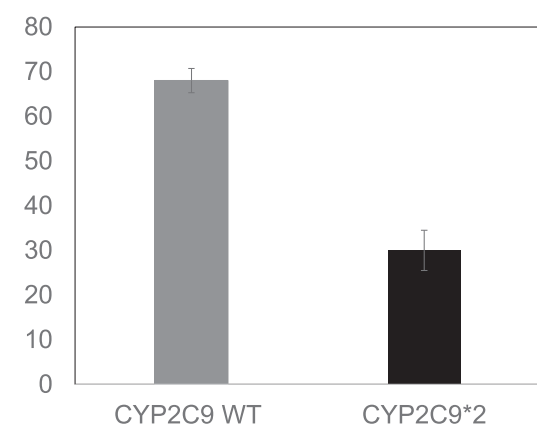

B

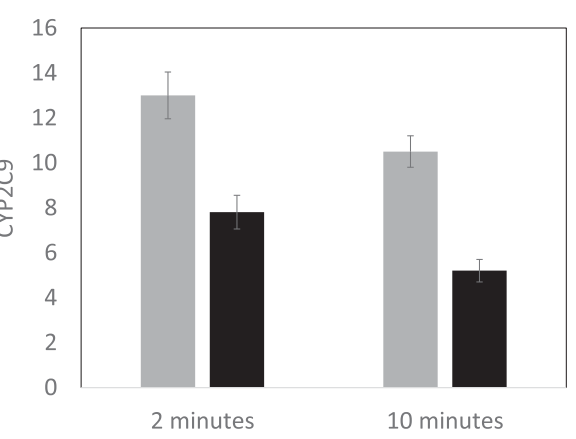

Fig. 8. (A) The amount of NADPH consumed (nanomole per minute per nanomole CYP2C9 WT or $* 2$ variant) in the presence of losartan at fixed concentration of $20 \mu \mathrm{M}$. (B) The hydrogen peroxide $\left(\mathrm{H}_{2} \mathrm{O}_{2}\right)$ produced after 2 and 10 minutes as determined using xylenol orange colorimetric assay is shown in gray for CYP2C9 WT and black for CYP2C $9 * 2$. in the loss of activity. Thus, in addition to the clear influence of genetic variation on the larger structural elements in the $* 2$ and $* 3$ complexes, the subtle changes in the structure seen in the * 30 complex may contribute to severely impaired activity toward various substrates of CYP2C9. Further studies are warranted to understand the role of side-chain reorientation and ligand interactions in the rare variant $* 30$ as well as in the more prevalent $* 2$ and *3 variants. Overall, the observed structural studies may help explain and corroborate the functional evidence of the significantly altered substrate hydroxylation profile characteristic of these CYP2C9 variants often referred to as "poor metabolizers."

It is notable that a water molecule was found near the heme iron within $\sim 3-4 \AA$ distance in several structures, including the CYP2C9 ligand-free (PDB 1OG2) and the losartan bound complexes of CYP2C9 WT (PDB 5XXI), CYP2C9*3 (PDB 5X24), and CYP2C9*30 (PDB 5X23), but not in the *2 complex. The orientation of losartan near the heme iron in the CYP2C9* 2 crystal structure is unproductive, consistent with that observed with the previously solved WT, CYP2C9*3, and CYP2C9*30 complexes. It is likely that the losartan reorients within the active site during catalysis to a more preferred or productive mode with imidazole ring closer to the heme iron consistent with the metabolic profile (Lo et al., 1995). However, the obtained pose in the crystal structure may suggest that such orientation is more favorable or amenable to crystallization, which is done in the absence of the redox partner cytochrome $\mathrm{P} 450$ reductase, and losartan could adopt multiple conformations in the large active site chamber. The computational docking studies clearly show the highest affinity pose of losartan as the one with the imidazole ring with hydroxyl closer or oriented toward the heme iron in the CYP2C9*2 active site. This pose was also observed in the CYP2C9 WT structure, indicating that the structures obtained using X-ray crystallography can accommodate multiple orientations of losartan in the active site. Whether binding of NADPHdependent cytochrome $\mathrm{P} 450$ reductase affects the orientation of ligand or plays an important role in reorienting the ligand in the active site to the metabolically favorable pose remains to be elucidated. From the structural studies, one of the important differences between the wild type and the *2 and *3 variants included the simultaneous binding of an additional losartan in the access channel, which may yield insights into the role of cooperativity or allosteric regulation. Nonetheless, the interaction and orientation of amino acid side chains could be as important as the binding of ligands or drugs with these enzymes. Overall, the distal substitution of an amino acid may result in rotation or changes of the neighboring amino acid side-chain conformations in nearby helices and affect the binding of the ligand in the access channel and the active site as seen in the *2 and *3 complexes, or it may alter the interactions with the bound ligand in the access channel as seen with the *30 complex.

To probe the effect of crystallization and crystal packing on the protein conformation, orientation, and occupancy of losartan, we retrospectively reviewed crystal growth conditions of other CYP2C9-losartan complexes and several crystal structures of the human CYP2C enzymes. The WT-losartan complex was crystallized in the absence of the facial amphiphile and differed considerably in the buffer components from the $* 30$-losartan complex, which was crystallized in the presence of the facial amphiphile (Maekawa et al., 2017). Yet, the two crystal structures were superimposable and contained three molecules of losartan in identical orientation. In contrast, the crystals of $* 3$ and $* 30$-losartan complexes grew in the presence of the facial amphiphile and with the same precipitant but slightly different buffer components. The *3 complex illustrated differences in the $\beta 4$ loop transducing from the Iso359Leu amino acid variation as well as the orientation and occupancy of losartan compared with the *30 and the WT complex. Despite these similarities and differences, the effect of ligand and buffer components, concentration, crystallization conditions, detergent molecules that include CYMAL-5 and/or facial amphiphiles, and the observed crystal packing cannot be ignored in the given crystallography work. Interestingly, the Arg144 residue is a highly conserved amino acid across all the human CYP2C enzymes along with many other CYP2C subfamily enzymes from different species that we analyzed using sequence alignment. As described in Supplemental Fig. 7A, the side chain of Arg144 in the CYP2C8 and the illustrated 2C9 structures (except the CYP2C9*2 complex with Arg144Cys variation) demonstrated hydrogenbonding interactions with the main chain atom of 261 . In addition, the only available CYP2C19 structure (PDB 4GQS) also illustrated similar but weak interactions between the side chain of Arg144 and main chain atom of 261. The residue at position 261 is glutamine in CYP2C9 and arginine in CYP2C8 and CYP2C19. Superimposing all the CYP2C8 and CYP2C19 structures with the CYP2C9 WT ligand-free, CYP2C9 WT, CYP2C9*3, and CYP2C9*2-losartan complexes clearly revealed the alignment of the F-G cassette and the G-H loop in all the structures except the current $* 2$ complex (Supplemental Fig. 7B). Many of these structures of the CYP2C enzymes were solved in various crystallization conditions and showed varied crystal packing, yet they superimposed well across the G-H loop and 
the F-G cassette, indicating the CYP2C9*2-losartan complex as an outlier in this region. Such analysis further suggests the role of distal variation while taking into consideration the impact of other variables.

To conclude, the structural information obtained yields insights into the loss of activity of losartan in patients with CYP2C9 variant alleles and further aids in understanding how protein structure influences protein function in biologic systems. The challenges remain because it is likely to observe a wide range of functional consequences among different substrates of CYP2C9, indicating that a substrate-dependent effect should be considered when assessing the outcome of genetic variations. In light of the above results that clearly demonstrate the differences in the active site between the variants and the WT, it is interesting to note the protective effect of $* 2$ and $* 3$ variants of CYP2C9 in limiting nonsmall cell lung cancer growth (Sausville et al., 2018). The patients possessing these CYP2C9 alleles demonstrated improved survival because of reduced epoxyeicosatrienoic acid synthesis and cell proliferation compared with those with the WT allele. More studies are needed to interpret the role of $* 2$ and $* 3$ as well as many rare variants of this important drug-metabolizing CYP2C9 enzyme in altering the response to drugs or exerting a protective role in disease progression. Investigations into the structurefunction relationships of genetic variants of P450 enzymes promise to reveal molecular determinants of drug binding that contribute to differences in dose response, which could lead to development of safer medications.

\section{Acknowledgments}

We thank the staff at Stanford Synchrotron Radiation Light source.

\section{Authorship Contributions}

Participated in research design: Parikh, Shah.

Conducted experiments: Parikh, Evans, Obi, Shah.

Contributed new reagents or analytic tools: Zhang, Maekawa.

Performed data analysis: Parikh, Evans, Obi, Glass, Shah.

Wrote or contributed to the writing of the manuscript: Parikh, Glass, Shah.

\section{References}

Bart AG and Scott EE (2017) Structural and functional effects of cytochrome $b_{5}$ interactions with human cytochrome P450 enzymes. $J$ Biol Chem 292 20818-20833.

Berman HM, Westbrook J, Feng Z, Gilliland G, Bhat TN, Weissig H, Shindyalov IN, and Bourne PE (2000) The protein data bank. Nucleic Acids Res 28:235-242.

Bumpus NN and Hollenberg PF (2010) Cross-linking of human cytochrome P450 2B6 to NADPH-cytochrome P450 reductase: identification of a potential site of interaction. J Inorg Biochem 104:485-488.

Chen VB, Arendall WB III, Headd JJ, Keedy DA, Immormino RM, Kapral GJ, Murray LW, Richardson JS, and Richardson DC (2010) MolProbity: all-atom structure validation for macromolecular crystallography. Acta Crystallogr D Biol Crystallogr 66:12-21.

Cohen AE, Ellis PJ, Miller MD, Deacon AM, and Phizackerley RP (2002) An automated system to mount cryo-cooled protein crystals on a synchrotron beam line, using compact sample cassettes and a small-scale robot. J Appl Cryst 35:720-726.

Crespi CL and Miller VP (1997) The R144C change in the CYP2C9*2 allele alters interaction of the cytochrome P450 with NADPH:cytochrome P450 oxidoreductase. Pharmacogenetics 7:203-210.

Cuff AL and Martin AC (2004) Analysis of void volumes in proteins and application to stability of the p53 tumour suppressor protein. J Mol Biol 344:1199-1209.

Daly AK, Rettie AE, Fowler DM, and Miners JO (2017) Pharmacogenomics of CYP2C9: functional and clinical considerations. J Pers Med 8:1.

Emsley P and Cowtan K (2004) Coot: model-building tools for molecular graphics. Acta Crystallogr D Biol Crystallogr 60:2126-2132.

Evans P (2006) Scaling and assessment of data quality. Acta Crystallogr D Biol Crystallogr 62:72-82.

Goldstein JA (2001) Clinical relevance of genetic polymorphisms in the human CYP2C subfamily. Br J Clin Pharmacol 52:349-355.

Guengerich FP, Waterman MR, and Egli M (2016) Recent structural insights into cytochrome P450 function. Trends Pharmacol Sci 37:625-640.
Ho PC, Abbott FS, Zanger UM, and Chang TK (2003) Influence of CYP2C9 genotypes on the formation of a hepatotoxic metabolite of valproic acid in human liver microsomes. Pharmacogenomics J 3:335-342.

Joy MS, Dornbrook-Lavender K, Blaisdell J, Hilliard T, Boyette T, Hu Y, Hogan SL, Candiani C, Falk RJ, and Goldstein JA (2009) CYP2C9 genotype and pharmacodynamic responses to losartan in patients with primary and secondary kidney diseases. Eur $J$ Clin Pharmacol 65:947-953.

Kleywegt GJ and Jones TA (1994) Detection, delineation, measurement and display of cavities in macromolecular structures. Acta Crystallogr D Biol Crystallogr 50: 178-185.

Lee SC, Bennett BC, Hong WX, Fu Y, Baker KA, Marcoux J, Robinson CV, Ward AB, Halpert JR, Stevens RC, et al. (2013) Steroid-based facial amphiphiles for stabilization and crystallization of membrane proteins. Proc Natl Acad Sci USA 110: E1203-E1211.

Leslie AG (1999) Integration of macromolecular diffraction data. Acta Crystallogr D Biol Crystallogr 55:1696-1702.

Lo MW, Goldberg MR, McCrea JB, Lu H, Furtek CI, and Bjornsson TD (1995) Pharmacokinetics of losartan, an angiotensin II receptor antagonist, and its active metabolite EXP3174 in humans. Clin Pharmacol Ther 58:641-649.

Long F, Vagin AA, Young P, and Murshudov GN (2008) BALBES: a molecularreplacement pipeline. Acta Crystallogr D Biol Crystallogr 64:125-132.

Maekawa K, Adachi M, Matsuzawa Y, Zhang Q, Kuroki R, Saito Y, and Shah MB (2017) Structural basis of single-nucleotide polymorphisms in cytochrome P450 C9. Biochemistry 56:5476-5480.

Maekawa K, Harakawa N, Sugiyama E, Tohkin M, Kim SR, Kaniwa N, Katori N, Hasegawa R, Yasuda K, Kamide K, et al. (2009) Substrate-dependent functional alterations of seven CYP2C9 variants found in Japanese subjects. Drug Metab Dispos 37:1895-1903.

McPhillips TM, McPhillips SE, Chiu HJ, Cohen AE, Deacon AM, Ellis PJ, Garman E, Gonzalez A, Sauter NK, Phizackerley RP, et al. (2002) Blu-Ice and the Distributed Control System: software for data acquisition and instrument control at macromolecular crystallography beamlines. J Synchrotron Radiat 9:401-406.

Miners JO and Birkett DJ (1998) Cytochrome P4502C9: an enzyme of major importance in human drug metabolism. Br J Clin Pharmacol 45:525-538.

Murshudov GN, Skubák P, Lebedev AA, Pannu NS, Steiner RA, Nicholls RA, Winn MD, Long F, and Vagin AA (2011) REFMAC5 for the refinement of macromolecular crystal structures. Acta Crystallogr D Biol Crystallogr 67:355-367.

Niinuma Y, Saito T, Takahashi M, Tsukada C, Ito M, Hirasawa N, and Hiratsuka M (2014) Functional characterization of 32 CYP2C9 allelic variants. Pharmacogenomics $J$ 14:107-114

Nourooz-Zadeh J, Tajaddini-Sarmadi J, and Wolff SP (1994) Measurement of plasma hydroperoxide concentrations by the ferrous oxidation-xylenol orange assay in conjunction with triphenylphosphine. Anal Biochem 220:403-409.

Ortiz de Montellano PR (2015) Structure, Mechanism, and Biochemistry, Springer, New York.

Potterton E, Briggs P, Turkenburg M, and Dodson E (2003) A graphical user interface to the CCP4 program suite. Acta Crystallogr D Biol Crystallogr 59:1131-1137.

Pravda L, Sehnal D, Toušek D, Navrátilová V, Bazgier V, Berka K, Svobodová Vareková R, Koca J, and Otyepka M (2018) MOLEonline: a web-based tool for analyzing channels, tunnels and pores (2018 update). Nucleic Acids Res 46 W368-W373.

Rettie AE and Jones JP (2005) Clinical and toxicological relevance of CYP2C9: drugdrug interactions and pharmacogenetics. Annu Rev Pharmacol Toxicol 45:477-494.

Rettie AE, Korzekwa KR, Kunze KL, Lawrence RF, Eddy AC, Aoyama T, Gelboin HV, Gonzalez FJ, and Trager WF (1992) Hydroxylation of warfarin by human cDNAexpressed cytochrome P-450: a role for P-4502C9 in the etiology of (S)-warfarindrug interactions. Chem Res Toxicol 5:54-59.

Sausville LN, Gangadhariah MH, Chiusa M, Mei S, Wei S, Zent R, Luther JM, Shuey MM, Capdevila JH, Falck JR, et al. (2018) The cytochrome P450 slow metabolizers CYP2C9*2 and CYP2C9*3 directly regulate tumorigenesis via reduced epoxyeicosatrienoic acid production. Cancer Res 78:4865-4877.

Shah MB, Liu J, Huo L, Zhang Q, Dearing MD, Wilderman PR, Szklarz GD, Stout CD, and Halpert JR (2016) Structure-function analysis of mammalian CYP2B enzymes using 7-substituted coumarin derivatives as probes: utility of crystal structures and molecular modeling in understanding xenobiotic metabolism. Mol Pharmacol 89:435-445.

Shah MB, Pascual J, Zhang Q, Stout CD, and Halpert JR (2011) Structures of cytochrome P450 2B6 bound to 4-benzylpyridine and 4-(4-nitrobenzyl)pyridine: insight into inhibitor binding and rearrangement of active site side chains. Mol Pharmacol 80:1047-1055.

Sica DA, Gehr TW, and Ghosh S (2005) Clinical pharmacokinetics of losartan. Clin Pharmacokinet 44:797-814.

Sim SC and Ingelman-Sundberg M (2013) Update on allele nomenclature for human cytochromes P450 and the Human Cytochrome P450 Allele (CYP-allele) Nomenclature Database. Methods Mol Biol 987:251-259.

Soltis SM, Cohen AE, Deacon A, Eriksson T, González A, McPhillips S, Chui H, Dunten P, Hollenbeck M, Mathews I, et al. (2008) New paradigm for macromolecular crystallography experiments at SSRL: automated crystal screening and remote data collection. Acta Crystallogr D Biol Crystallogr 64:1210-1221.

Sullivan-Klose TH, Ghanayem BI, Bell DA, Zhang ZY, Kaminsky LS, Shenfield GM, Miners JO, Birkett DJ, and Goldstein JA (1996) The role of the CYP2C9-Leu359 allelic variant in the tolbutamide polymorphism. Pharmacogenetics 6:341-349.

Trott O and Olson AJ (2010) AutoDock Vina: improving the speed and accuracy of docking with a new scoring function, efficient optimization, and multithreading. $J$ Comput Chem 31:455-461.

Van Booven D, Marsh S, McLeod H, Carrillo MW, Sangkuhl K, Klein TE, and Altman RB (2010) Cytochrome P450 2C9-CYP2C9. Pharmacogenet Genomics 20:277-281.

Wei L, Locuson CW, and Tracy TS (2007) Polymorphic variants of CYP2C9: mechanisms involved in reduced catalytic activity. Mol Pharmacol 72:1280-1288. 
Wilderman PR, Shah MB, Jang HH, Stout CD, and Halpert JR (2013) Structural and thermodynamic basis of (+)- $\alpha$-pinene binding to human cytochrome P450 2B6. $J$ Am Chem Soc 135:10433-10440.

Williams PA, Cosme J, Ward A, Angove HC, Matak Vinković D, and Jhoti H (2003) Crystal structure of human cytochrome P450 2C9 with bound warfarin. Nature 424:464-468.

Winn MD, Ballard CC, Cowtan KD, Dodson EJ, Emsley P, Evans PR, Keegan RM, Krissinel EB, Leslie AG, McCoy A, et al. (2011) Overview of the CCP4 suite and current developments. Acta Crystallogr D Biol Crystallogr 67:235-242.
Yasar U, Tybring G, Hidestrand M, Oscarson M, Ingelman-Sundberg M, Dahl ML, and Eliasson E (2001) Role of CYP2C9 polymorphism in losartan oxidation. Drug Metab Dispos 29:1051-1056.

Address correspondence to: Manish B. Shah, Department of Pharmaceutical Sciences, Albany College of Pharmacy and Health Sciences, 106 New Scotland Ave., Albany, NY 12208. E-mail: manish.shah@acphs.edu 